\title{
Strain-Mediated Asymmetric Growth of Plasmonic Nanocrystals: A Monometallic Au Nanorod-Au Nanoparticle Heterodimer.
}

\author{
Yihan Zhu ${ }^{1}$, Jianfeng Huang ${ }^{1}$, Yu Han ${ }^{1}$ \\ 1. Advanced Membranes and Porous Materials Center, Physical Sciences and Engineering Division, \\ King Abdullah University of Science and Technology (KAUST), Thuwal 23955-6900, Kingdom of \\ Saudi Arabia
}

Colloidal noble metal nanocrystals (NCs) usually exhibit highly symmetric particle morphologies, as dictated by their intrinsic crystallographic symmetries. Breaking this morphological symmetry would bring vast variations to the plasmonic and optical properties of NCs, greatly enriching their applications in plasmonics, nanophotonics, sensing, and surface-enhanced Raman scattering (SERS). However, the synthesis of monometallic dimers via seed-mediated growth is difficult, because one of the main driving forces for dimerization is the lattice mismatch between two materials. When the growth material is the same as the seed, epitaxial growth on the entire surface of the seed particle is favored over the formation of a dimer. ${ }^{1}$ On the other hand, most reported dimers are constituted of two particles, whereas dimers with alternate configurations, for instance, a nanoparticle (NP) grown on a single-crystalline nanorod (NR) with a regular morphology, are yet to be explored. Given the high degree of symmetry of metallic structures, it is conceivable that growing a single NP on a highly regular NR would be difficult because it would require one site on the NR at which the NP is grown to be differentiated from many other symmetryequivalent sites.

Here, we report that by employing specific strong ligands (thiols), an asymmetric AuNR-AuNP dimer can be synthesized from single crystalline AuNR seeds. Using electron microscopy and tomography, we reconstructed the three-dimensional morphology of the dimer crystal and identified that the newly grown AuNP is a multiple-twinned crystal preferentially residing at the "neck" region of the AuNR. The welldefined morphology of single crystalline AuNRs (with known surface facet indices) allows us to easily identify the formation of a dimer interface and to track the subsequent evolution of the AuNPs. The results provide important insights into the growth mechanism of this dimeric nanostructure, suggesting that the NP first nucleates at one (111) bridging facet of the NR, which further grows as a consequence of random twinning, and finally recrystallizes into a single multiple-twinned crystal.

We found the key factors for successful synthesis include inhomogeneous surface strains on the AuNR and appropriate reduction kinetics, which are both associated with the use of ligands. A mechanism was proposed that describes the growth of AuNP in the dimer structure as follows. The chemisorption of ligands induces inhomogeneous strains on the surface of AuNR. Among different surface facets of the AuNR, the (111) bridging facets at the rod's "neck" are the most strained and are thus preferred locations for $\mathrm{Au}$ deposition to form a twinning structure via stacking faults that can largely relieve the surface strain. $^{2}$ Once the first twin structure is formed on a (111) facet, this facet is differentiated from other symmetry-equivalent facets, becoming an "active" site for the further growth of Au through successive twinning, as driven by the same strain-relieving mechanism. New twins can form randomly in different $<111>$ directions, resulting in various agglomerates of small grains. Worm-like structures are formed when most new grains grow laterally along the rod, while radiative twinning leads to cauliflower-like structures. At the final stage of synthesis, the agglomerates undergo a recrystallization process with small grains fused into large ones, accompanied by the disappearance of grain boundaries. As a consequence, 
different-shaped agglomerates gradually develop into single AuNPs with similar shapes. If the reaction is prolonged, the NPs have fewer grains and smoother surfaces.

The key hypothesis of our proposed mechanism is that thiol ligands induce significant and inhomogeneous surface strain on the AuNR. In order to verify this hypothesis, we used HRTEM to characterize the assynthesized AuNR (with weaker ligand CTAB) and the AuNR incubated with thiol, and then we visualized their strain distributions using geometric phase analysis (GPA). Atomic-resolution HRTEM images were taken for both types of AuNR along the [110] axes, as the displacement of the densely packed (111) layers could be identified in this direction (Figures 1a, c). Interestingly, the GPA analysis showed that unlike the as-synthesized AuNR that exhibited little strain fluctuation over the entire crystal (Figure 1b), the thiol-incubated AuNR exhibited large shear deformation on the rod surface that was particularly localized on the (111) bridging facets in the "neck" regions (Figure 1d). Aligning the y-axis of the GPA map with the growth direction (i.e., the [001] axis) of the AuNR, the shear strain fields $\left(\varepsilon_{x y}\right)$ in left and right bridging facets have opposite signs, indicating the same type of shear deformation caused by gliding of the $\{111\}$ planes (Figure 1d). The shear strain was determined to be from the $<112>\{111\}$ slip. In the Bragg-filtered HRTEM image of the thiol-incubated AuNR, an array of rhombuses are delineated by connecting neighboring atomic columns (Figures 1e, f). The magnitudes of the strains in different areas can be intuitively identified from the obtuse angles $(\theta)$ of the rhombus. Specifically, the angles measured at the side surface of the rod body $\left(\theta: 108.1^{\circ}-109.9^{\circ}\right)$ are very close to the value for a perfectly ABC-stacked bulk structure $\left(\theta=109.6^{\circ}\right)$ (Figure 1f), whereas apparently smaller angles $\left(\theta: 102.5^{\circ}-104.3^{\circ}\right)$ are observed at the bridging facets (Figure 1e). These results clearly demonstrate that thiols can selectively induce large surface strain in the "neck" region of the AuNR, where the surface (111) planes undergo shear deformation along the $[11 \overline{2}]$ direction.

\section{References:}

[1] Sohn, K.; Kim, F.; Pradel, K. C.; Wu, J.; Peng, Y.; Zhou, F.; Huang, J. ACS Nano 3 (2009), 2191.

[2] Paine, D. C.; Howard, D. J.; Stoffel, N. G. J. Electron. Mater. 20 (1991), 735.

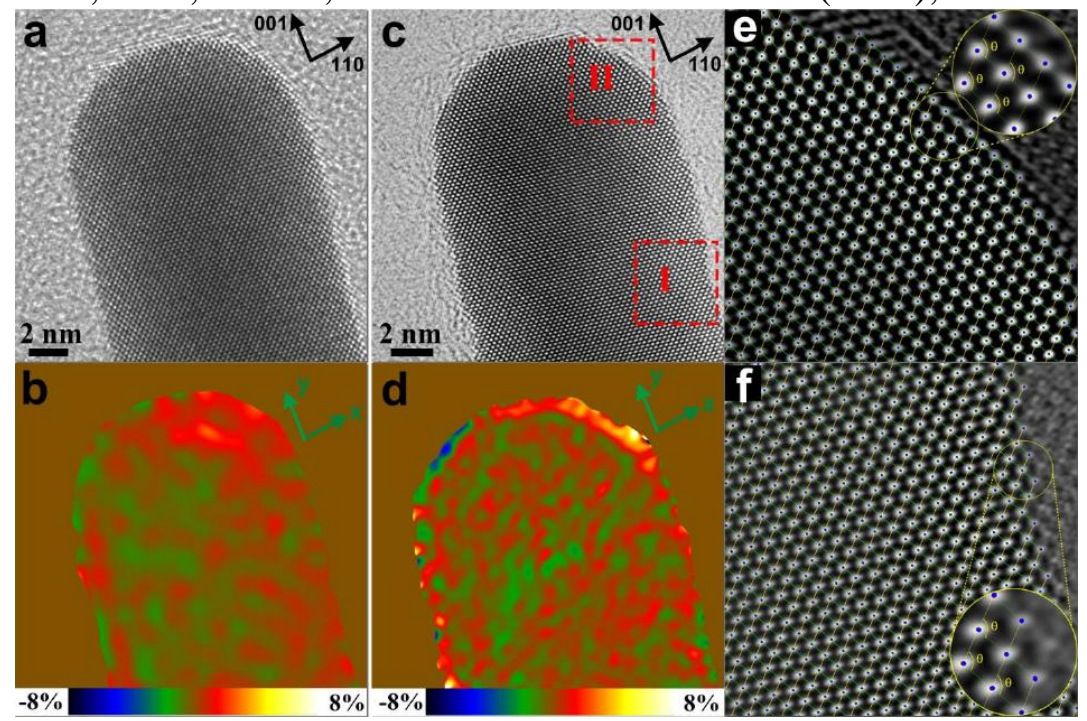

Figure 1. (a, c) Atomic-resolution HRTEM images of (a) the as-synthesized (CTAB capped) AuNR and (c) thiol-incubated AuNR taken along the [1 $\overline{10}]$ axes. (b, d) corresponding strain distributions of the shear component ( $\varepsilon_{x y}$, the magnitude cut-off is $\pm 8 \%$ ) determined by geometric phase analysis. (e, f) Enlarged Bragg-filtered HRTEM images of (f) region I and (e) region II, as marked in (c). 